\title{
Variación estacional de la apendicitis aguda
}

\author{
Hubert James Mendoza-Rojas ${ }^{1}$, José Julio Valle Bayona ${ }^{2}$
}

\section{RESUMEN}

Objetivo: Conocer si aumentaron los casos de apendicitis agudas atendidos en las estaciones de verano y primavera durante 2005-2014 en el Hospital de Emergencias Grau de Lima.

Material y Métodos: Estudio observacional, transversal y retrospectivo. Muestreo no probabilístico, tamaño de la muestra de 6,917 operados por apendicitis aguda entre los años 2005 - 2014 en el Hospital de Emergencias Grau de Lima. Se utilizó una ficha de recolección de datos de historias clínicas elaborándose una base de datos. Se aplicó test de análisis de varianzas de apendicetomías y correlación entre temperaturas y número de apendicitis.

Resultados: La edad mínima y máxima fue de 14 y 93 años. El 52.6\% $(3,639)$ de los casos fueron de sexo femenino. En enero se realizó el mayor número mensual de apendicectomías, con 648, 634 en marzo y 616 en febrero. El Análisis de Varianza obtuvo un valor $p$ de 0.24 , no hubo diferencias significativas entre las estaciones del año. Hubo correlación parcial entre temperatura mensual y número de apendicectomías.

Conclusiones: Existe un patrón estacional con aumento de la frecuencia de la apendicitis aguda en verano y primavera, y un descenso en otoño e invierno. El aumento de casos de apendicitis aguda es del $3.32 \%$ y $3.75 \%$ en verano con respecto a otoño e invierno, sin diferencia estadística significativa entre las cuatro estaciones del año.

Palabras clave: apendicitis, apendicectomía, estacional, verano.

\section{Seasonal variation of acute appendicitis}

\section{ABSTRACT}

Objective: To know if there was an increase in acute appendicitis cases treated in summer and spring among 2005-2014 in Grau Emergency Hospital in Lima.

Material and Methods: Observational, cross-sectional and retrospective study. Non-probabilistic sampling, sample size of 6,917 operated on for acute appendicitis during 2005-2014 in the Grau Emergency Hospital in Lima. We used a form for data collection from medical records to develop a database. Analysis of variance test was applied to appendectomies numbers and correlation between temperature and number of appendicitis.

Results: The minimum and maximum age was 14 and 93 years old. $52.6 \%(3,639)$ of the cases were female. January was the highest monthly number of appendectomies, with 648; 634 in March and 616 in February. The analysis of variance obtained a $\mathrm{p}$ value $=0.24$, there was no significant difference among the seasons of the year. There was a partial correlation between monthly temperature and number of appendectomies.

Conclusions: There is a seasonal pattern with increase in the frequency of acute appendicitis during summer and spring, and a decline in autumn and winter. The increase in cases of acute appendicitis is 3.32\% and $3.75 \%$ in summer compared to autumn and winter, with no significant statistical difference among the four seasons of the year.

Key words: appendicitis, appendectomy, seasonal, summer.

1. Cirujano General Asistente del Servicio de Cirugía General del Hospital III de Emergencias Grau (EsSalud). Lima, Perú.

2. Médico Ocupacional, Docente de Posgrado de la Facultad de Medicina Humana, USMP. 


\section{INTRODUCCIÓN}

La apendicitis aguda ocurre frecuentemente en adultos jóvenes (1), aunque puede presentarse a cualquier edad (2), siendo la prevalencia puntual a lo largo de varios periodos de aproximadamente el $7 \%(3)$.

Como factores de riesgo se incluyen sexo masculino, hábitos alimentarios, susceptibilidad familiar, estación invernal, presencia de agentes obstructivos (4-7).

La variación estacional documentada en áreas específicas como factor de riesgo, no es frecuente en la literatura mundial. En estudios realizados en pacientes adultos, desde países con altas temperaturas (8-11) en verano como Pakistán (12), hasta aquellos países con bajas temperaturas como Finlandia (13), se describe un incremento de la frecuencia de la apendicitis aguda en los meses de verano y primavera y una disminución en otoño e invierno $(14,15)$.

En los estudios realizados en niños, se ha descrito que la mayor incidencia de apendicitis es en primavera, porque en esta estación se incrementan los casos de infecciones virales, postulándose que ello condicionaría la formación de hiperplasia linfática, lo que facilitaría la obstrucción del lumen apendicular (16). Otro estudio en la India, sin embargo, señala que no existiría variación estacional y la apendicitis aguda es más frecuente en estaciones lluviosas con gran humedad y con presencia de alérgenos (17).

Un estudio en Nigeria describe que la mayor frecuencia de casos de apendicitis ocurre en épocas lluviosas (18) proponiéndose la hipótesis de su asociación con alérgenos del polen.

En Turquía, en un estudio realizado en dos ciudades con diferentes altitudes y características climáticas, la apendicitis aguda fue más frecuente en verano en la ciudad de menos altitud, comparada con otra ciudad ubicada a mayor altitud, donde la apendicitis fue más frecuente en invierno (19).

En el Perú, en un estudio ecológico de grupos múltiples se encontró una correlación positiva entre la temperatura y la tasa de incidencia de apendicitis (20).

En este estudio se desea comprobar si el aumento de la frecuencia de casos de apendicitis agudas atendidas en el Servicio de Cirugía General del Hospital de Emergencias Grau, sigue la variación estacional reportada en varios estudios especialmente en las estaciones de verano y primavera en el periodo 2005-2014.

\section{MATERIAL Y MÉTODOS}

Se diseñó un estudio observacional de corte transversal y retrospectivo. El estudio se realizó en el Servicio de Cirugía General del Hospital III Emergencias Grau (EsSalud), ubicado en el Cercado de Lima.

La unidad de análisis fue el paciente operado por apendicitis aguda con edad igual y mayor a 14 años. Se realizó un muestreo no probabilístico por conveniencia de tipo accidental, considerando a todos los pacientes operados por apendicitis aguda y registrada en la oficina de estadística del Hospital Grau.

El instrumento de recolección de datos fue una ficha de datos con alternativas cerradas, utilizando la técnica de inspección de registros de datos de estadística e historias clínicas para la elaboración de una base de datos.

El tamaño de la muestra fue de 6,917 pacientes operados por apendicitis aguda entre los años 2005 2014. Los criterios de inclusión de los pacientes fueron: edad igual y mayor a 14 años de edad, con diagnóstico histológico de apendicitis aguda, historia clínica del paciente con datos completos requeridos en la ficha de datos y los criterios de exclusión fueron, pacientes menores de 14 años de edad, sin diagnóstico histológico de apendicitis aguda, tener datos incompletos en la historia clínica.

Se consideró asimismo el registro de las temperaturas diarias y promedio mensual obtenidas en la estación meteorológica 846280 (SPIM) del Aeropuerto Internacional Jorge Chávez en Lima, ubicada en Latitud: -12, Longitud: -77.11, Altitud: 12 msnm para el periodo 2005-2014, administrada por la Oficina de Climatología de Corpac S.A.

Se realizó el procesamiento con tabulación, construcción de tablas y gráficos mediante el programa de Microsoft Excel 2010. Se realizó el análisis de datos con pruebas como el test de análisis de varianzas para determinar la diferencia significativa de las prevalencias entre las estaciones del año durante el periodo de 2005-2014. 


\section{RESULTADOS}

En el Servicio de Cirugía General del Hospital III Emergencias Grau durante el periodo 2005-2014 se registraron 6,917 apendicectomías por apendicitis aguda. El rango de edad mínima y máxima fue de 14 a 93 años. El sexo femenino representó la mayoría de los casos con 3,639 casos (52.6\%) y el masculino con $3,278(47.4 \%)$. La muestra incluida en el estudio fue de 6,917 pacientes.
La tabla 1, muestra la distribución de frecuencia de las apendicectomías por meses y año. De los 10 años estudiados, el mes de enero tiene la mayor frecuencia de apendicectomías en 4 años, le siguen febrero en 2 años, marzo en 2 años, julio en 1 año y noviembre en 1 año.

Tabla 1. Distribución de frecuencia mensual de las apendicectomías, periodo 2005-2014

\begin{tabular}{|c|c|c|c|c|c|c|c|c|c|c|c|c|c|}
\hline \multirow[t]{2}{*}{ Año } & \multicolumn{12}{|c|}{ Meses } & \multirow[t]{2}{*}{ Total } \\
\hline & Enero & Febrero & Marzo & Abril & Mayo & Junio & Julio & Agosto & Setiembre & Octubre & Noviembre & Diciembre & \\
\hline 2005 & 46 & 47 & 43 & 43 & 45 & 37 & 34 & 47 & 29 & 40 & 49 & 45 & 505 \\
\hline 2006 & 48 & 60 & 34 & 43 & 32 & 36 & 49 & 59 & 32 & 54 & 51 & 35 & 533 \\
\hline 2007 & 61 & 56 & 59 & 43 & 47 & 39 & 46 & 49 & 43 & 55 & 48 & 49 & 595 \\
\hline 2008 & 67 & 60 & 58 & 62 & 52 & 55 & 59 & 53 & 42 & 57 & 62 & 50 & 677 \\
\hline 2009 & 64 & 55 & 75 & 58 & 63 & 64 & 69 & 75 & 59 & 61 & 46 & 66 & 755 \\
\hline 2010 & 66 & 64 & 74 & 72 & 64 & 61 & 43 & 55 & 39 & 62 & 64 & 62 & 726 \\
\hline 2011 & 76 & 59 & 69 & 68 & 62 & 55 & 64 & 53 & 59 & 53 & 79 & 67 & 764 \\
\hline 2012 & 68 & 59 & 77 & 66 & 67 & 68 & 57 & 73 & 63 & 57 & 60 & 53 & 768 \\
\hline 2013 & 81 & 80 & 71 & 70 & 58 & 65 & 63 & 80 & 71 & 62 & 68 & 70 & 839 \\
\hline 2014 & 71 & 76 & 74 & 61 & 62 & 50 & 61 & 66 & 46 & 73 & 61 & 54 & 755 \\
\hline Total & 648 & 616 & 634 & 586 & 552 & 530 & 545 & 610 & 483 & 574 & 588 & 551 & 6917 \\
\hline
\end{tabular}

Con relación al total de meses por todos los años, en el mes de enero con 648 siendo el mayor número de apendicectomías, seguido de 634 realizadas en el mes de marzo y luego el mes de febrero con 616. El año 2013 presentó el mayor número de apendicectomías con 839.
La figura 1, nos muestra un patrón de curvas de las frecuencias de apendicitis aguda que acentúa descensos en el primer semestre, incrementos los meses de julio y agosto con decremento en setiembre y recuperación hacia el final del año; tendencia observada en los 10 años de estudio.

Figura 1. Distribución de frecuencia de apendicectomías por año y mes 2005-2014

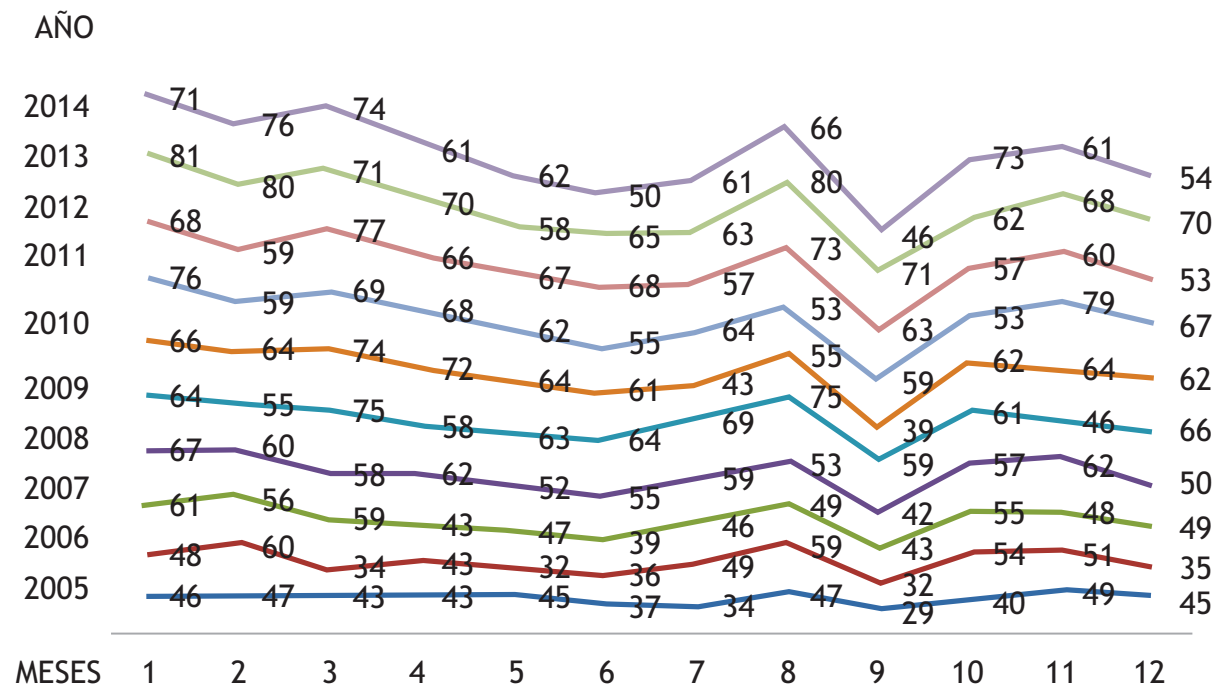


La figura 2, muestra el canal endémico en el periodo 2005-2014 basado en los percentiles 25, 50 y 75, con tendencias de zonas de alarma y seguras más bajas hacia los meses considerados de la estación de invierno y su elevación hacia los meses de verano.

Figura 2. Canal endémico de apendicectomías por mes 2005-2014

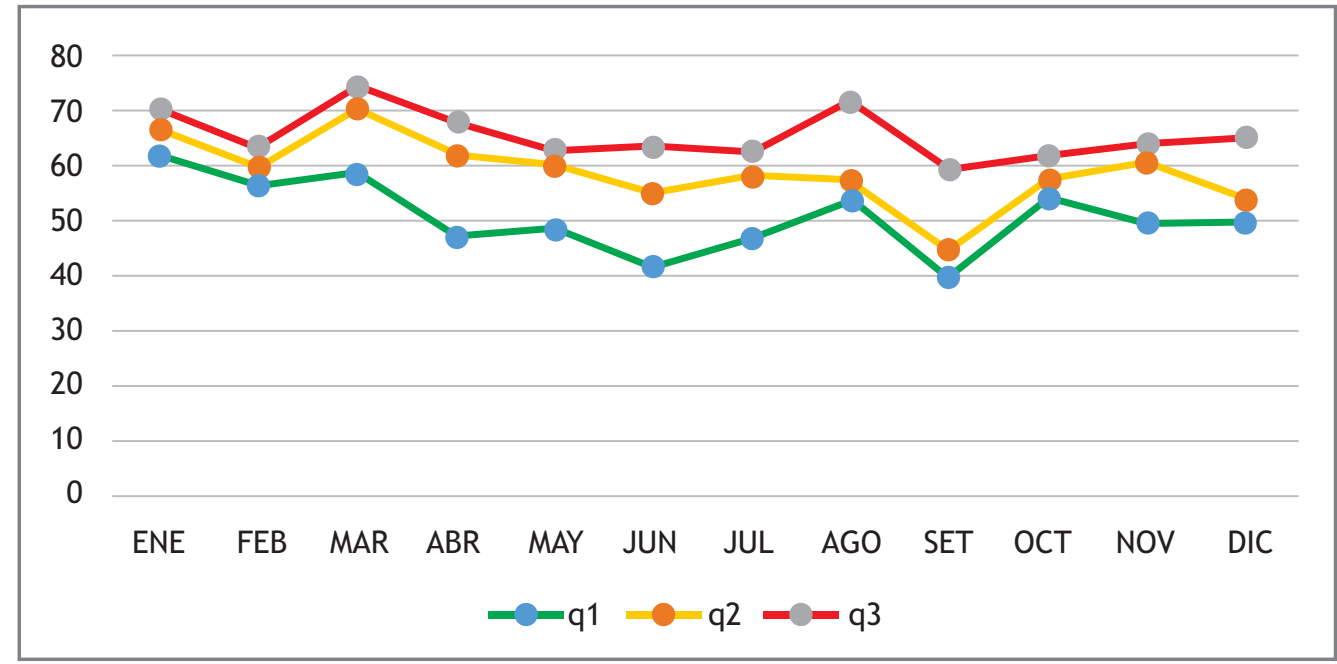

Considerando la asignación de meses en el año a estaciones (verano: diciembre 22 - marzo 21; otoño: marzo 22 - junio 21; invierno: junio 22 - setiembre 22; primavera: setiembre 23 - diciembre 21).

La tabla 2, muestra la distribución de las apendicectomías según la estación del año. De los 10 años de estudio, en 9 años durante el verano se produjeron el mayor número de apendicitis aguda. Siendo el total de apendicectomías durante el verano en los 10 años de 1898.

Tabla 2. Distribución de frecuencia de las apendicectomías según año y estación

\begin{tabular}{|c|c|c|c|c|c|}
\hline & \multicolumn{5}{|c|}{ Estaciones } \\
\hline & Verano & Otoño & Invierno & Primavera & \\
\hline 2005 & 136 & 125 & 110 & 134 & 505 \\
\hline 2006 & 142 & 111 & 140 & 140 & 533 \\
\hline 2007 & 176 & 129 & 138 & 152 & 595 \\
\hline 2008 & 185 & 169 & 154 & 169 & 677 \\
\hline 2009 & 194 & 185 & 203 & 173 & 755 \\
\hline 2010 & 204 & 197 & 137 & 188 & 726 \\
\hline 2011 & 204 & 185 & 176 & 199 & 764 \\
\hline 2012 & 204 & 201 & 193 & 170 & 768 \\
\hline 2013 & 232 & 193 & 214 & 200 & 839 \\
\hline 2014 & 221 & 173 & 173 & 188 & 755 \\
\hline Total & 1898 & 1668 & 1638 & 1713 & 6917 \\
\hline
\end{tabular}


La figura 3, nos muestra como la frecuencia de las apendicitis agudas llega a su pico más alto en el

verano y desciende en otoño e invierno, para luego ascender en la primavera.

Figura 3. Frecuencia de apendicectomías según año y estación, periodo 2005-2014

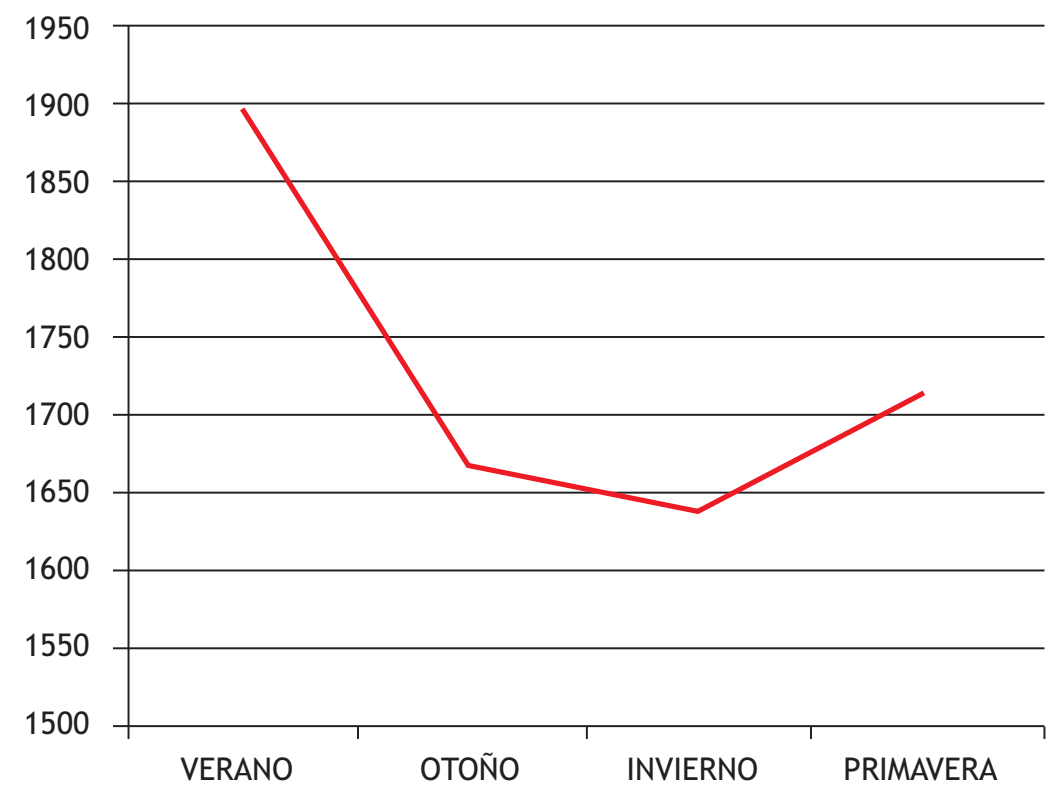

La figura 4, presenta la variación de temperatura meses de enero, febrero y marzo y menores en los mensual promedio registrada en Lima en el mismo meses de agosto y setiembre.

periodo 2005-2014, mostrando las mayores en los

Figura 4. Temperatura promedio mensual en Lima periodo 2005-2014

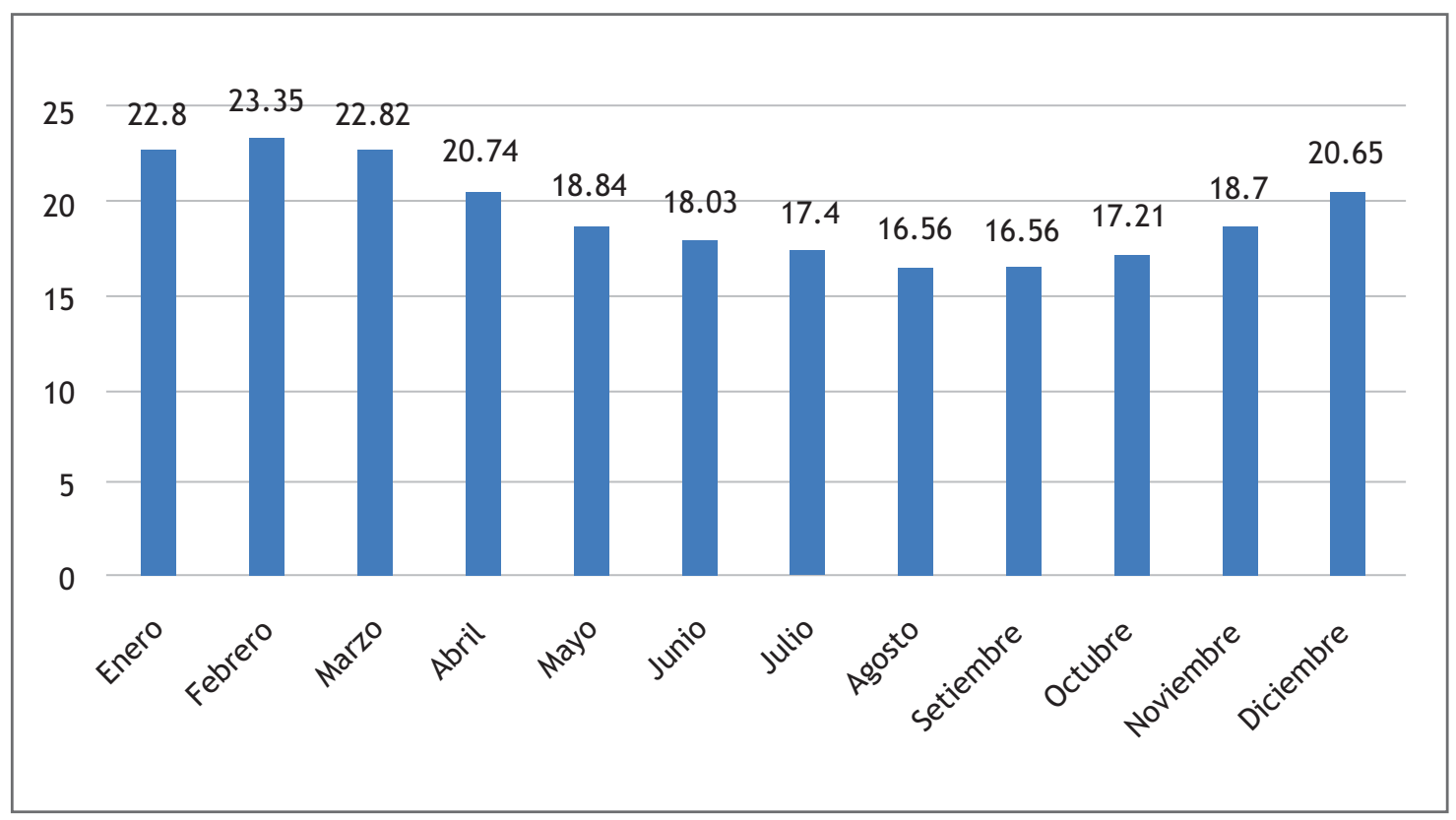


La tabla 3, nos muestra el promedio del total de apendicectomías en el periodo 2005-2014 de acuerdo a las estaciones del año, el verano presenta 189.8 apendicectomías, otoño 166.8 , invierno 163.8 y primavera 171.3 apendicectomías.

Tabla 3. Promedio de apendicectomías según estación

\begin{tabular}{|ccc|}
\hline Estación del año & $\begin{array}{c}\text { Número de apendicitis } \\
(2005-2014)\end{array}$ & $\begin{array}{c}\text { Promedio anual de } \\
\text { apendicitis por estación }\end{array}$ \\
\hline Verano & 1898 & 189.8 \\
\hline Otoño & 1668 & 166.8 \\
\hline Invierno & 1638 & 163.8 \\
\hline Primavera & 1713 & 171.3 \\
\hline
\end{tabular}

En la figura 5, observamos también la tendencia del el descenso en otoño e invierno. promedio de apendicectomías, de un pico en verano y

Figura 5. Curva de promedios según estación

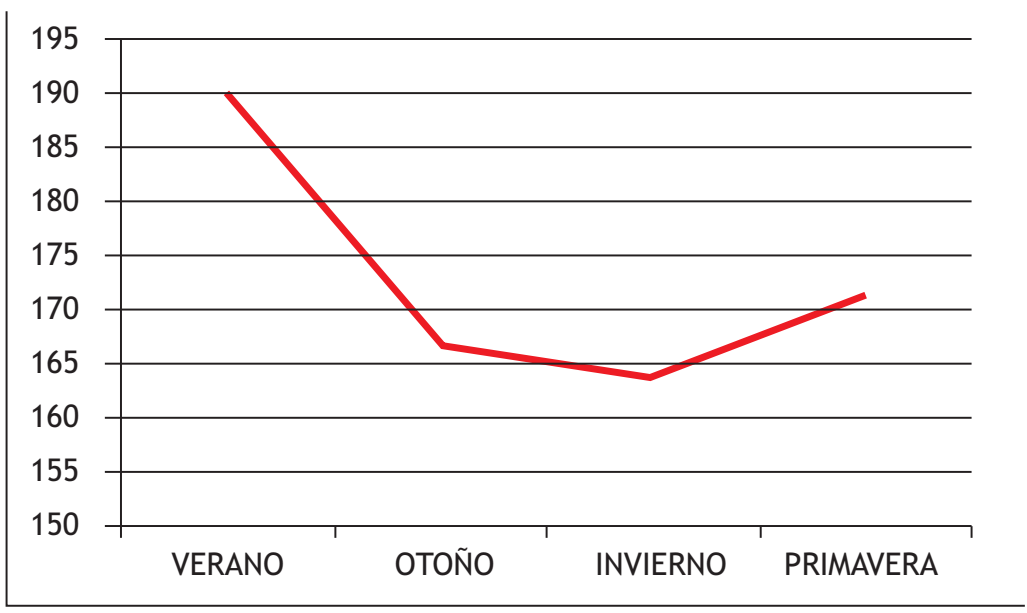

La tabla 4, muestra que el aumento de los casos de respecto a las estaciones de otoño e invierno. apendicitis en verano es del $3.32 \%$ y $3.75 \%$ con

Tabla 4. Número de apendicectomías y porcentajes según estación

\begin{tabular}{|ccc|}
\hline Estación & $\begin{array}{c}\mathbf{N}^{\circ} \text { de apendicitis } \\
(2005-2014)\end{array}$ & Porcentaje \\
\hline Verano & 1898 & $27.43 \%$ \\
\hline Otoño & 1668 & $24.11 \%$ \\
\hline Invierno & 1638 & $23.68 \%$ \\
\hline Primavera & 1713 & $24.76 \%$ \\
\hline Total & 6917 & $100 \%$ \\
\hline
\end{tabular}

El análisis de varianza de un factor para comprobar si existía diferencia significativa entre los valores absolutos observados en las 4 estaciones del año, con un nivel de significancia del $5 \%(0,05)$ y un intervalo de confianza para la media del $95 \%$, obtuvo el valor $p$ de 0.241 , siendo mayor que el valor alfa $(0,05)$, lo que se muestra en la tabla 5. 
Tabla 5. Resultado del Análisis de varianza

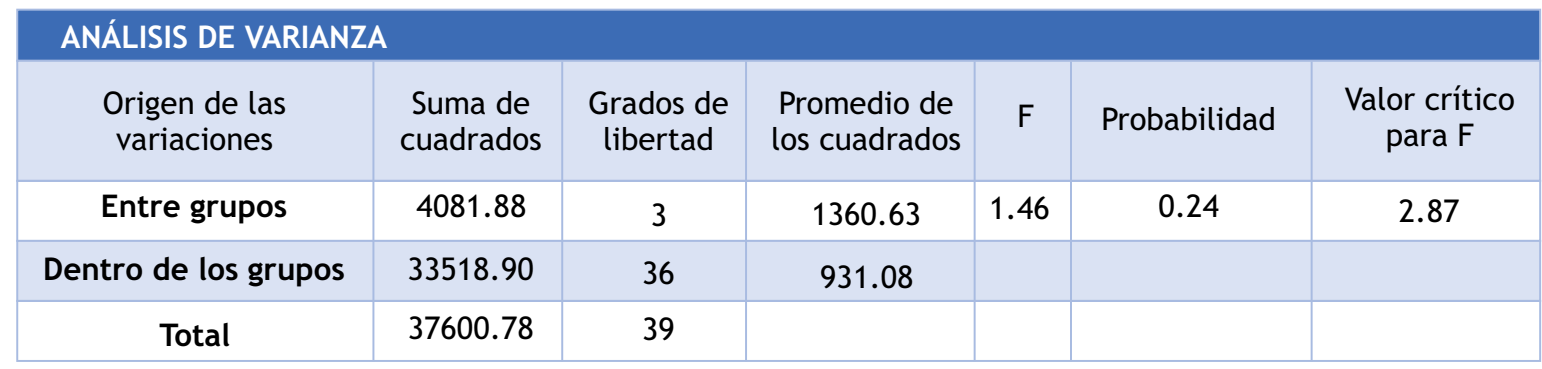

\begin{tabular}{|lcccc|}
\hline \multicolumn{1}{|c}{ Rrupos } & Cuenta & Suma & Promedio & Varianza \\
\hline Verano & 10 & 1898 & 189.8 & 974.4 \\
\hline Otoño & 10 & 1668 & 166.8 & 1084.8 \\
\hline Invierno & 10 & 1638 & 163.8 & 1120.4 \\
\hline Primavera & 10 & 1713 & 171.3 & 544.7 \\
\hline
\end{tabular}

La tabla 6, presenta los valores de apendicectomías totales ocurridas en el periodo y se compara con el promedio mensual de temperatura en el mismo periodo. El coeficiente de determinación $r 2$ fue de 0.67 , mostrando una correlación aceptable, es decir el $67 \%$ de las variaciones en el número de apendicectomías se debió a la temperatura comparada. El coeficiente $\mathrm{r} 2$ aplicado a los meses de enero a julio fue 0.95 , y en el periodo setiembre a diciembre de 0.42 .

Tabla 6. Apendicectomías y temperatura promedio mensual 2005-2014

\begin{tabular}{|c|c|c|c|c|c|c|c|c|c|c|c|c|}
\hline Variables & Enero & Febrero & Marzo & Abril & Mayo & Junio & Julio & Agosto & Setiembre & Octubre & Noviembre & Diciembre \\
\hline Apendicectomías & 648 & 616 & 634 & 586 & 552 & 530 & 545 & 610 & 483 & 574 & 588 \\
\hline Temperatura ${ }^{\circ} \mathrm{C}$ & 22.8 & 23.35 & 22.82 & 20.74 & 18.84 & 18.03 & 17.4 & 16.56 & 16.56 & 17.21 & 18.7 & 20.65 \\
\hline
\end{tabular}

\section{DISCUSIÓN}

Los resultados obtenidos en la presente investigación, concuerdan con los resultados obtenidos en la mayoría de trabajos alrededor del mundo, en que se evidencia un aumento de la frecuencia de apendicitis aguda en la estación de verano.

Fares (21) realizó una revisión sistemática de 11 trabajos de investigación de 8 países, sobre los patrones estacionales de la apendicitis aguda, desde 1970 al 2012, y encontró que la apendicitis aguda era más frecuente en verano en la mayoría de los países, a excepción de dos trabajos de investigación de Nigeria y Turquía que encontraron que fue en invierno.
Las razones de estas tendencias estacionales son inciertas, pero se ha reportado varios factores que podrían jugar un papel importante, como los diferentes virus o bacterias que causan infecciones a diferentes temperaturas, el efecto de alérgenos en los meses calurosos, la humedad, la polución, la dieta baja en fibra, etc. Y que se evidencia más este patrón estacional en las ciudades con poca altitud y cercanas al mar (19), algo característico de la ciudad de Lima. Pero hasta hoy en día no hay una causa específica atribuida a los cambios de clima (22).

Cuando realizó el análisis de varianza de los cuatro grupos estacionales en el presente trabajo de investigación, para determinar si existía una diferencia significativa entre las 4 estaciones, no se evidenció una diferencia significativa. 
Y esto también lo describe Wolkomir (23), quien estudió 12,686 apendicectomías y encontró un aumento de la incidencia de apendicitis en las estaciones de primavera y verano, sin embargo al realizar el análisis de varianza no encontró una variación estadísticamente significativa.

Sin embargo en el estudio de las correlaciones, éstas obtuvieron valores importantes excepto en el mes de agosto donde hubo un aumento ligero pero no significativo de las apendicectomías, no encontrando en el presente estudio una causa probable.

Existen varios factores que condicionan la apendicitis aguda y uno de ellos podría ser las estaciones de verano y primavera, no constituyendo un factor determinante.

En conclusión, existe un patrón estacional con aumento de la frecuencia de la apendicitis aguda en verano y primavera, y un descenso en otoño e invierno. El aumento de casos de apendicitis aguda es del $3.32 \%$ y $3.75 \%$ en verano con respecto a otoño e invierno. Asimismo, no existe una diferencia estadística significativa del número absoluto de apendicectomías entre las cuatro estaciones del año; sin embargo existe un comportamiento correlacionado del número de apendicectomías total en el periodo de estudio con las temperaturas mensuales registradas.

\section{REFERENCIAS BIBLIOGRÁFICAS}

1. Brunicardi FC, Andersen DK, Billiar TR, et al, eds. The appendix. In: Schwartz's principles of surgery. 9th ed. New York, NY: McGraw-Hill; 2012:2043-2067.

2. Itskowitz MS, Jones SM. Appendicitis. Emerg Med. 2004; 36:10-1.

3. Koepsell TD. In search of the cause of appendicitis. Epidemiology. 1991; 2:319-321.

4. Olakolu S, Lloyd C, Day G, Wellington P. Diagnosis Of Acute Appendicitis At Mandeville Regional Hospital (MRH): Clinical Judgment Versus Alvarado Score. The Internet Journal of Surgery. $2010: 27: 1$.

5. Andersson N, Griffiths H, Murphy J, Roll J, Serenyi A, Swann I, et al. Is appendicitis familial? Br Med J 1979; 2: 697-8.

6. Adamidis D, Roma-Giannikou E, Karamolegou K, et al. Fiber intake and childhood appendicitis. Int J Food Sci Nutr. 2000; 51:153-157.

7. Pieper R, Kager L, Weintraub A, Lindberg AA, Nord CE. The role of Bacteroides fragilis in the pathogenesis of acute appendicitis. Acta Chir Scand 1982; 148: 39-44.

8. Rai R. An Evaluation of the Seasonal Variation in Acute Appendicitis. J Evol Med Dent Sci. 2014;3(2):257-60.

9. Noudeh $\mathrm{Y}$, Sadigh N, Ahmadnia A. Epidemiologic features, seasonal variations and false positive rate of acute appendicitis in Shahr-e-Rey, Tehran. Int J Surg 2007, 5(2):95-98
10. Al-Omran M, Mamdani M, MacLeod R. Epidemiologic features of acute appendicitis in Ontario, Canada. Can J Surg 2003;46(4):263-268

11. Lin KB. Epidemiology of appendicitis and appendectomy for the low-income population in Taiwan, 2003-2011. BMC Gastroenterology 2015;15:18

12. Khan M, Naz S, Zarin M, Rooh-ul-Muqim, Salman M. Epidemiological observations on appendicitis in Peshawar, Pakistan. Pak J Surg 2012; 28(1):30-33

13. Ilves I. Seasonal variations of acute appendicitis and nonspecific abdominal pain in Finland. World J Gastroenterol 2014;20(14):4037-4042

14. Gallerani M, Boari B, Anania G, Cavallesco G, Manfredini R. Seasonal variation in onset of acute appendicitis. Clin Ter 2006;157(2):123-127.

15. Wei PL, Chen CS, Keller JJ, Lin HC. Monthly variation in acute appendicitis incidence: A 10-year nationwide populationbased study. J Surg Res 2012;178(2):670-676

16. Pepper VK. Diagnosis and Management of Pediatric appendicitis, intussusception, and Meckel diverticulum. Surg Clin NAm 2012;92:505-526.

17. Jangra B, Jangra MS, Rattan K N, Kadian YS. Seasonal and day of week variations in acute appendicitis in north Indian children. J Indian Assoc Pediatr Surg 2013;18:42-3

18. Oguntola AS, Adeoti ML, Oyemolade TA. Appendicitis: Trends in incidence, age, sex, and seasonal variations in SouthWestern Nigeria. Ann Afr Med 2010;9(4):213-7

19. Sulu B, Günerhan, Palanci Y, Ișler B, Çağlayan K. Epidemiological and demographic features of appendicitis and influences of several environmental factors. Ulus Travma Acil Cerrahi Derg 2010;16(1):38-42

20. Tejada-Llacsa P, Melgarejo-García G. Incidencia de apendicitis aguda y su relación con factores ambientales, Perú, 2013. An Fac med. 2015;76(3):253.

21. Fares, A. Summer appendicitis. Annals of Medical and Health Sciences Research 2014;4(1):18-21

22. Gul T, Khan M, Sahar S, Kamran W, Andaleeb U. Epidemiological and demographic features of appendicitis and influences of several environmental factors. Pak J Surg 2014; 30(3):245-247

23. Wolkomir A, Kornak P, Elsakr M, McGovern P. Seasonal variation of acute appendicitis: a 56-year study. South Med J 1987;80(8):958-60.

\section{Fuentes de financiamiento}

Este trabajo ha sido financiado por los autores.

\section{Conflictos de interés}

Los autores declaran no presentar ningún conflicto de interés.

\section{Correspondencia:}

Hubert James Mendoza Rojas

Dirección: Avenida Grau N 351, Cercado de Lima.

Hospital de Emergencias Grau. Servicio de Cirugía General.

Teléfono: 992758186

Correo electrónico: hubertjames86@hotmail.com 